\title{
The active commissioning process for a power reactor spent fuel reprocessing pilot plant in China
}

\author{
ZHANG TianXiang*, WANG Jian, WU Tao, CHEN GuangJun, DI WU YongQing \& \\ RU FaQuan
}

The 404 Company Limited, China National Nuclear Corporation, Lanzhou 732850, China

Received April 16, 2011; accepted May 31, 2011

\begin{abstract}
The process of a power reactor spent fuel reprocessing pilot plant (hereinafter referred to as the "pilot plant") had been completed through active commissioning. Operational and technological parameters, such as shearing, dissolution, feed clarification, co-decontamination cycle, uranium and plutonium purification cycle, and the uranium and plutonium finishing facility, were identified. In addition, technical devices including extraction and mechanical equipment, electrical installation as well as instrumentation, and auxiliary systems for safety and adaptability were also verified. The commissioning results indicated that the recovery rate and decontamination coefficients of each system satisfied the designed index requirements and the qualified productions, i.e. uranium trioxide and plutonium dioxide, were produced. Monitored values at various monitoring points in the radiological protection system were within the control range and the discharge of waste water and waste gas complied with the relevant standards. This shows that independent and innovative technology for power reactor spent fuel reprocessing had been developed by our country.
\end{abstract}

spent fuel reprocessing, pilot plant, commissioning, recovery rate, purification

Citation: Zhang T X, Wang J, Wu T, et al. The active commissioning process for a power reactor spent fuel reprocessing pilot plant in China. Chinese Sci Bull, 2011, 56: 2411-2415, doi: 10.1007/s11434-011-4600-2

After nearly seven years of constant effort and through water commissioning, acid commissioning, cold uranium commissioning, followed by $5 \%, 50 \%$ and $100 \%$ active commissioning in succession, the active commissioning of a Chinese pilot plant for spent fuel reprocessing was successfully completed on December 21, 2010, using independent and innovative technology.

After many years of scientific research experiment and engineering construction, the commissioning was formally carried out in the China reprocessing facility in 2004. Water commissioning and acid commissioning as well as cold uranium commissioning were carried out successively in a co-decontamination cycle, uranium purification cycle, plutonium purification cycle, uranium finishing facility and plutonium finishing facility. The process, mechanical equip-

\footnotetext{
*Corresponding author (email: cgjwhut@163.com)
}

ment, electrical installation and instrumentation were also validated during the commissioning described above, obtaining much important data during water commissioning, acid commissioning and cold uranium commissioning. Subsequently, problems revealed during commissioning were rectified and finally active commissioning, consisting of three stages, $5 \%, 50 \%$ and $100 \%$ active commissioning, were completed.

At present, commercial reprocessing plants in operation or that have been operated once at large scale, such as French UP2 and UP3 plants [1,2], the British THORP plant [3], and the American West Valley plant [4], all have years of operational experience. The active commissioning at POKKASHO reprocessing plant was carried out in March, 2006 [5]. Compared with the advanced reprocessing technology in operation abroad, partial procedure of China's spent fuel reprocessing technology needs to be further developed. 
This article mainly introduces the active commissioning situation for a power reactor spent fuel reprocessing pilot plant in China. The active commissioning success indicates that we have already grasped the related key technologies, which will provide the important platform for China's advanced reprocessing engineering technology. Meanwhile it has also raised a specialized talent team for China's reprocessing.

\section{Active commissioning}

\subsection{Tasks for active commissioning}

(i) To verify the operational performance of the system and obtain operational parameters for radioactive feed by shearing and dissolution of spent fuel elements, settling centrifuge and feed preparation;

(ii) To acquire the operational parameters during the active commissioning stage; and to verify whether or not the operational performance of extraction equipment in the chemical separation section and the recovery rate, decontamination coefficient and uranium and plutonium separation coefficients in each system could satisfy the designed index;

(iii) To verify and choose the operational conditions and safe performance index of the medium-level liquid waste evaporator in the active commissioning stage;

(iv) To ensure that the key equipment in the uranium finishing facility, i.e. uranium concentration evaporator, electrolyzer and fluidized bed, could operate stably to meet the technological requirements;

(v) To verify whether or not the technical process in the plutonium finishing facility, i.e. oxalate plutonium precipitation, filtration, dry roasting, etc., could meet the technological requirements;

(vi) To ensure that the solid waste could be stored safely and temporarily, and the discharge of effluents complied with the relevant management requirements;

(vii) To validate the operational function of the radiological protection system during the 5\%,50\% and $100 \%$ active commissioning and to confirm its safety, effectiveness, and obtain the necessary radiological protection data;

(viii) To check the adaptability of equipment and facilities, such as mechanical equipment and electrical installation as well as instrumentation, etc., during the active commissioning.

\subsection{Goal of active commissioning}

To master the technical process for active commissioning through shearing a given number of spent fuel elements, and validate the adaptability, safety and reliability of the technical process and equipment. Furthermore, to optimize and adjust the operational and technological parameters, and finally to produce qualified uranium and plutonium.

\section{Results and discussion}

\subsection{Process system}

(i) Head end. Using a shearing machine researched and developed independently in China, based on previous shearing experiments on simulated fuel elements, a real fuel element was sheared. A tentative adjustment of shearing parameters was carried out and the equilibrium between shearing and dissolution was optimized during the active commissioning to ensure a smooth dissolution process and confirm the final shearing parameters. The short fuel element sheared was then dissolved in a dissolver, in which the negative pressure and feed temperature were controlled within certain range. For a given feed rate and centrifugal separation speed, the sedimentation and clarification (separation between liquid and solid) during dissolution were carried out using a decanter centrifuge designed and developed independently in China. The residual, settled in the drum walls, was washed by water at a specified centrifuge rotation speed and after detecting and analyzing, the resultant water was merged into 1AW (aqueous phase from the 1A column) and then sent to be stored temporarily in high-level liquid waste tank.

After attaining the separation of liquid and solid, the dissolution was converted into $1 \mathrm{AF}$ feed, which consisted of dissolution and cold uranium at the appropriate volume ratio during $5 \%$ and $50 \%$ active commissioning phases and of dissolution during the $100 \%$ active commissioning. The qualified $1 \mathrm{AF}$ feed was subjected to precise metering through the balance tank in order to meet the requirement of mass balance.

The shearing machine, dissolver and settling centrifuge operated well throughout active commissioning, indicating that the equipment in the head end (researched and developed independently in China), was successfully applied to spent fuel reprocessing, filling in a domestic gap in this field.

(ii) Co-decontamination separation cycle. The main tasks of the co-decontamination separation cycle were to achieve co-extraction and co-decontamination of uranium and plutonium, strengthen the removal of technetium, and to separate the uranium and plutonium. The density and interface of key extraction equipment in the co-decontamination separation cycle, i.e. the $1 \mathrm{~A}$ column (uranium and plutonium co-extraction and co-decontamination column), the TcS column (washing column for technetium removal), the 1B column (uranium and plutonium separation column) and the 1C column (uranium reextraction column), could be controlled within the set range and the interface of the mixersettler was maintained steadily.

The uranium and plutonium recovery rate, decontamination coefficient and separation coefficient in the codecontamination cycle were able to meet the technological index requirement during the active commissioning. 1A column, in particular, could operate stably and continously, 
and the recovery rate of uranium and plutonium and the decontamination coefficient satisfied the technical index. The radioactivity decontamination coefficient of $1 \mathrm{~A}$ column was several times above the design value, resulting in a greatly reduced burden on the following purification process.

Both the hydraulics performance and mass-transfer performance in the co-decontamination separation cycle were able to satisfy the technological requirements.

(iii) Uranium purification cycle. The main tasks of the uranium purification cycle were to remove the plutonium, ruthenium and neptunium, and to achieve further purification of uranium. The key extraction equipments for the uranium purification cycle were a 2D mixer-settler (for extracting uranium in the second cycle), a 2E column (uranium re-extraction in the second cycle), a 3D column (uranium extraction in the third cycle) and a $3 \mathrm{E}$ column (uranium re-extraction in the third cycle). During the active commissioning, the interface of the 2D mixer-settler and pulse column could be steadily maintained, and the density of the pulse column mentioned above could be controlled within the set range.

The uranium recovery rate and decontamination coefficient of the uranium purification cycle met the technical index requirements during active commissioning. The burden to the uranium purification cycle was greatly reduced because the decontamination coefficient for the codecontamination cycle was far above the design value. As a result, using 2EU (aqueous phase containing uranium from the $2 \mathrm{E}$ column), the uranium trioxide product prepared by denitration satisfied the product quality requirement.

(iv) Plutonium purification cycle. The main tasks of the plutonium purification cycle were to remove the uranium, zirconium and niobium, and achieve further purification and concentration of plutonium. The key pieces of equipment for the second cycle plutonium purification cycle were a $2 \mathrm{~A}$ column (plutonium extraction), a 2B column (plutonium reextraction) and a degreasing column. The density and interface between these could be steadily maintained and controlled within the set range, respectively. During the cold uranium commissioning stage, the extraction equipments were tested with cold uranium in place of cold "plutonium" and the technical conditions determined tentatively laid a reliable foundation for the following active commissioning.

The plutonium recovery rate and decontamination coefficient for the plutonium purification cycle met the technological requirements during active commissioning. Reflux extraction technology was feasible and plutonium concentration of 2 BP (aqueous phase containing plutonium from the 2B column) met the requirements for the oxalate precipitation.

(v) Uranium finishing facility. The aqueous phase feed containing uranium from the uranium purification cycle entered the uranium concentration evaporator for evaporation and concentration. The resultant concentrated uranium had two paths for entry (density controlled). One path entered the electrolyzer for the preparation of uranous by elec- trolyzing uranium and the other path entered the fluidized bed for the preparation of uranium trioxide production by denitrification. During active commissioning, the temperature and pressure were kept constant and the density and liquid level were controlled within the range of technological requirements when the uranium concentration evaporator was operated at the specified operating parameters.

During the $50 \%$ and $100 \%$ active commissioning, after the rectification of the cell body, the uranous concentration coming from the electrolyzer reached the designed value, which was based on the optimized technological and operational parameters obtained from previous experiments, while the total uranium and hydrazine concentrations as well as the acidity were all within the design value range, satisfying the technological requirements of the uraniumplutonium separation in the $1 \mathrm{~B}$ system.

The heating system, nozzle and cowl of the fluidized bed had been optimized and rectified based on the previous cold uranium commissioning. During 5\%, 50\% and $100 \%$ active commissioning, the denitration temperature and feed rate as well as the pressure, flow rate and temperature of the fluidizing gas, atomizing gas and back-flushing gas, were all within the controllable range for the fluidized bed. Uranium trioxide was produced, as a result of compliance of the total uranium content, radioactivity level and main impurities contents with relevant standards.

(vi) Plutonium finishing facility. The feed containing plutonium from the plutonium purification cycle entered the plutonium finishing facility for precipitation, filtering and dry roasting in order, in which the temperature and negative pressure were kept constantly. Plutonium dioxide was produced, as a result of compliance of the plutonium content, radioactivity level and main impurities contents with relevant standards. In the cold uranium commissioning stage, simulation verification was carried out using uranyl nitrate solution rather than four-valent plutonium solution, and the technological parameters of the technical process, such as precipitation, filtering and roasting, were determined tetatively, which laid a reliable foundation for the smooth operation during active commissioning.

\subsection{Sampling and analyzing system}

During active commissioning, several thousand samples were received in the analysis section and up to ten thousand analytical data points were obtained and quoted. Generally speaking, sampling and transfer equipment, as well as the analytical instruments, operated normally. The analytical data checked and quoted complied with quality guarantee requirements and the operating environment conformed to the safety requirements for radiological protection.

The sampling system adopted the method of air lift with a vacuum auxiliary to achieve the collection of radioactive feed. We initially faced difficulties with regard to sampling and a lack of representative samples. These problems were 
solved through a series of rectifications. The key equipment for a pneumatic sample transfer system, i.e. a transceiver, commutator, cap opener and so on, which were researched and developed independently, run reliably, developing the domestic manufacture of a radioactive pneumatic sample transfer system.

\subsection{Radiological protection system}

After the confirmation of the active commissioning, radiation monitoring systems operated well, and the operation of major monitoring equipment, such as for critical, neutron and gaseous effluents, remained stable. The monitoring systems included monitoring of aerosol and nitrogen oxides, as well as I-129/Kr-85 in process offgas, which were rectified or re-built after the $5 \%$ active commissioning, were able to operate normally and satisfy the radiation monitoring demand during the active commissioning. In the workplace, the management and regulatory measures for radiological safety were effective and the radiological level was less than the regulatory standards. The personal doses were monitored after entering the control field, and the maximal individual dose in a single work period was 273 $\mu \mathrm{Sv}$ during active commissioning, which was lower than the allowed value of $1 \mathrm{mSv}$. The regulatory measures were reasonable.

Monitoring the situation of regional radiation levels: the average irradiation dose in the green zone was in the range of $1.32-2.33 \mu \mathrm{Sv} / \mathrm{h}$, which was less than the allowed value of $5 \mu \mathrm{Sv} / \mathrm{h}$, and in the range of $1.83-14.8 \mu \mathrm{Sv} / \mathrm{h}$ in the orange area, which was lower than the allowed value of 25 $\mu \mathrm{Sv} / \mathrm{h}$, indicating that the controlled external radiation level status was in good condition. The negative pressure gradients were established in various areas, and the gas direction was correct, satisfying the designed operational index (controlled values of negative pressure: green area, 20-40 Pa; orange area, 40-60 Pa; equipment room, 100-150 Pa; chamber, 200-300 Pa). The monitoring data of the special neutron monitoring system showed that the technology operated stablely, and the critical accident alarm system remained normal. The values at the various monitoring points mentioned above were far below the set alarm limits. Both the aerosol level and surface contamination in workshop were monitored according to plan, and the monitoring values were all lower than the limit value.

\section{4 “Three wastes" systems}

In order to reduce the volume of waste liquid, the mediumlevel liquid waste need to be treated by evaporation, during which the destruction of nitric acid with formaldehyde could adopt intermittent or continuous operation. During active commissioning, the former was the main style, and the evaporator operated stably and the key parameters, such as the negative pressure, liquid level, were controlled in the set range. Thus the evaporator safety guaranteed by them could be controlled during the evaporation process. The liquid effluents were discharged in a type of tank and carried out to sample and analyze before discharging, and the resultants were lower than the limits. The systems of both exhaust air in workshop and technological exhaust operated normally, and decontamination coefficients were higher than the designed value.

During active commissioning, the radionuclides, for instance, uranium, plutonium, in the liquid waste produced at high-and medium-levels, were analyzed and monitored by batch, and after satisfying the monitoring requirement, the waste liquids were then sent to be stored in private facilities temporarily.

During active commissioning, solid wastes were classified to collect, pack, mark, and store temporarily after monitoring according to the requirements, and the production and management of them were in controlled stage. Solid wastes mainly included the hulls undissolved, filter core, protective gloves, respirators, plastic sheeting, decontamination articles and so on.

\subsection{Other systems}

The auxiliary equipment and systems, such as for reagent preparation, water, electricity, wind, compressed air, steam, communication, fire control, and physical protection, operated well and could satisfy the operational requirements safely and stably, which ensured the success of the active commissioning process.

\section{Conclusions}

During active commissioning, the technological systems operated stably. A massive amount of data was accumulated for each procedure and the control and operational parameters of various equipments were obtained during stable operation. Qualified uranium trioxide and dioxide plutonium productions were achieved upon completion. Through active commissioning, the pilot plant process was mastered and the adaptability of the radiological protection system, mechanical equipment and electric installation, as well as instrumentation, etc., were validated and comprehensively confirmed:

(i) The shearing machine, dissolver and decanter centrifuge, etc. in the head end operated smoothly, and the preparation and precise metering of the $1 \mathrm{AF}$ feed satisfied the technological requirements;

(ii) The equipment in chemical separation cycle, such as the pulse column, mixer-settler, pulse generators, air-lift systems and metering pump, operated stably, and the recovery rate of uranium and plutonium, decontamination coefficient and separation coefficient were able to satisfy the designed index. In particular for the $1 \mathrm{~A}$ column, the radioac- 
tivity decontamination coefficient was several times above the design value, resulting in a greatly reduced burden in the subsequent purification process;

(iii) The evaporator, fluidized bed for denitration, precipitation reactor, filter, roasting oven, weighing and packaging facilities, and monitoring pollution equipments in the uranium and plutonium finishing facility operated stably, and measuring accuracy satisfied the mass balance and radiological protection requirements, and qualified uranium and plutonium production were produced finally;

(iv) The various effluents were in controlled stage, and ensured to be discharged, which were lower than the limits. The radioactive waste liquid and solid wastes were classified to collect and stored safely and temporarily;

(v) The operation of mechanical equipment, electrical installation, and instrumentation were in good condition overall and could meet the continuous and stable technological operation requirements; (vi) The radiological protection system operated normally and the control measures for radiological safety were effective. The radioactive pollution level and personal dosage exposure in the workplace were controlled well.

1 Emin J L, Pechard C, Tribout-Maurizi A, et al. AREVA NC experience of industrial scale MOX treatment in UP2-800. In: Proceedings of Global Conference, Pairs, France, 2009. 9024

2 Debreuille M F. Reprocessing at La Hauge: Industrial experience and perspectives. In: Proceedings of Global Conference, New Orleans, USA, 2003. 119

3 Schneider M, Coeytaux X, Faid Y B, et al. Possible toxic effects from the nuclear reprocessing plants at Sellafield and Cap de la Hague. Scientific and Technological Options Assessment, European Parliament, 2001. 11

4 Rippon S. Reprocessing-What went wrong? Nucl Eng Inter, 1976, 24: $21-27$

5 Sugiyama H, Nago T, Ishihara N, et al. Summary of active test at ROKKASHO reprocessing plant. In: Proceedings of Global Conference, Pairs, France, 2009. 9190

Open Access This article is distributed under the terms of the Creative Commons Attribution License which permits any use, distribution, and reproduction in any medium, provided the original author(s) and source are credited. 